\title{
Pérdidas necesarias e innecesarias: El duelo del analista ${ }^{1}$
}

\author{
Sandra Buechler ${ }^{2}$ \\ New York, USA
}

En este trabajo la autora reflexiona sobre las pérdidas que afronta el clínico y el significado y la elaboración del duelo por parte del terapeuta/analista, de la experiencia y el trabajo vivido con el paciente, así como de sí mismo en su calidad de profesional ejerciente. Tras definir el duelo y revisarlo desde el punto de vista de la teoría de las emociones, remite a la experiencia vivida por ella con la muerte de una paciente, y el impacto tanto valioso como doloroso de esta experiencia. Aborda igualmente las pérdidas por interrupciones y abandonos de tratamiento, y las de su finalización. Subraya la importancia de no negar la pérdida real, lo insustituible de la otra persona, sino de incorporar la experiencia a nuestro bagaje experiencial y clínico.

Palabras clave: Duelo, Pérdida, Psicoterapeuta, Psicoanalista.

In this work, the author reflects on the losses faced by the clinician and the meaning and the elaboration of grief on the part of the therapist / analyst, of the experience and work lived with the patient, as well as of himself in his/her capacity as a practicing professional. After defining grief and reviewing it from the point of view of the theory of emotions, she refers to her experience with the death of a patient, and the valuable and painful impact of this experience. It also addresses losses due to treatment interruptions and abandonments, and those of its termination. The author underlines the importance of not denying the real loss, the irreplaceable of the other person, but of incorporating the experience into our experiential and clinical baggage.

Key Words: Mourning, Grief, Psychotherapist, Psychoanalyst

English Title: Necessary and Unnecessary Losses: The Analyst's Mourning.

\section{Cita bibliográfica / Reference citation:}

Buechler, S. (2021). Pérdidas necesarias e innecesarias: El duelo del analista. Clínica e Investigación Relacional, 15 (1): 11-27. [ISSN 1988-2939] [Recuperado de www.ceir.info ] DOI:

10.21110/19882939.2021.150101

\footnotetext{
${ }^{1}$ Este trabajo es una versión revisada del incluido en la parte II, capítulo 5, de la obra de la autora: "Psychoanalytic Reflections, Training and Practice" (Astoria, NY: International Psychoanalytic Books, 2017), que a su vez es una revisión y ampliación de un trabajo publicado originalmente como: Buechler, S. (2000). Necessary and Unnecessary Losses. The Analyst'Mourning, Contemporary Psychoanalysis, 36(1):77-90. Traducción castellana de Mario Nervi Vidal y Alejandro Ávila. Reproducido con autorización de la autora @

2 Sandra Buechler, Ph.D. (New York), actualmente jubilada, ha sido Psicoanalista, Analista Didacta y Supervisora en el William Alanson White Institute y supervisora en el Programa postdoctoral y de Residentes del Columbia Presbyterian Hospital, así como en el Institute for Contemporary Psychotherapy. Entre sus obras: Valores de la Clínica: Emociones que guían el tratamiento psicoanalítico (2004); Marcando la diferencia en las vidas de los pacientes: Experiencia emocional en el ámbito terapéutico (2008); Permanezco ejerciendo: Las alegrías y angustias de una carrera clínica (2012); Comprendiendo y tratando pacientes en Psicoanálisis Clínico: Lecciones desde la Literatura (2015); Reflexiones psicoanalíticas. Formación y práctica (2018) y La aproximación psicoanalítica a los problemas del vivir (2019). Varias de ellas han sido traducidas al castellano y publicadas en la colección Pensamiento Relacional (Madrid: Ágora Relacional).
} 


\section{Introducción}

He dedicado al Duelo al menos un capítulo en cada una de mis obras (2004, 2008, 2012, 2015) ${ }^{1}$. Obviamente, este tema tiene una gran resonancia personal. Pero esa no es la única razón de mis muchas vueltas sobre este tema. Creo que mis puntos de vista sobre el duelo, que han evolucionado a lo largo de los años, son un aspecto importante de mi perspectiva analítica interpersonal.

Este artículo es un intento temprano de definir lo que implica el duelo, desde mi punto de vista. Al releerlo, todavía escucho ideas heredadas de mis días como teórica de las emociones. Aplico algunos conceptos teóricos, extraídos de las teorías de la emoción, a la situación de los analistas que soportan las pérdidas de sus pacientes.

¿Cuáles son las pérdidas evitables e inevitables que sufre un analista? Tras una revisión de varias concepciones del duelo, sugiero algunas complicaciones específicas en el proceso de duelo del analista, dependiendo de cómo ocurra la pérdida, y describo mi propia experiencia de la muerte de un paciente, incluyendo aspectos de mi duelo que (aún) están en curso. Escribir este artículo me ayudó a entender lo que me ayudó a conocerla, como analista y como ser humano. Esto de alguna manera modificó la experiencia de perderla. "Cada vez que la recuerdo me ayuda de alguna manera a cambiar el equilibrio entre lo que gané de ella y lo que sufrí".

Pero me tomaría varios años más (2015, p.150 de la v.0. [2008]) desarrollar mi propia visión interpersonal de los aspectos más dolorosos del duelo. "Creo que puede ser tentador el crear una versión saludable del duelo que domestique sus agonías y no haga justicia a nuestra necesidad de vivir a los demás como palpables, encarnados e impredecibles, y no solo sus características ... una verdadera comprensión de nuestras relaciones interpersonales. La naturaleza nos obliga a concluir que la pérdida de otros reales, específicos e íntimamente conocidos es insustituible. Necesitamos sentir su respiración, tomar su mano, verlos reír, experimentarlos en el momento vivido, a través de todos los sentidos, y no sólo en el recuerdo ... No importa lo madura y bien desarrollada que sea nuestra vida interior, no hay objeto real que sustituya a una pareja viva cuando se quiere ir a bailar".

\section{Pérdidas necesarias e innecesarias: El duelo del analista}

Nos encontrábamos en lo que me parecía ser una fase particularmente productiva del tratamiento. Por fin (desde mi punto de vista) la paciente era capaz de reconocer aspectos narcisistas de su personalidad que habían obstaculizado la posibilidad de mantener cualquier relación duradera. Ella tenía treinta y cinco años. Yo me sentía realmente preparada, tal vez 
por primera vez en todo lo que llevábamos de tratamiento, para acceder a un nivel de intimidad más profundo. Toda su vida había sido una auténtica lucha valiente y descomunal con su discapacidad física. Por fin, parecía lista para emerger con plenitud desde las constricciones de sus limitaciones psicológicas.

Fue durante un fin de semana cuando recibí el mensaje de que había muerto repentinamente.

Mi experiencia ante esta pérdida fue dolorosa en extremo, pero sé que no soy la única que ha pasado por este tipo de experiencias. ¿Cómo somos capaces de soportar estas circunstancias? Una vez pasado el tiempo, ¿Qué repercusión tienen en nuestra vida a nivel personal y profesional?

Es verdad que la pérdida de un paciente suele ser mucho menos dramática que el ejemplo que expongo. Los pacientes suelen finalizar el tratamiento, a veces de forma abrupta, en otras ocasiones tras una preparación meditada, pero ¿de qué manera impacta la repetida experiencia de tantos finales a lo largo de los años? ¿Acaso hay alguna forma mejor de soportar las penas que nos acompañan a lo largo de nuestra peculiar vida profesional?

Al describir algunas particularidades de la relación analítica, especialmente en su fase de finalización, Nina Coltart (1996) escribe:

La finalización definitiva, que parece ir contra corriente de todo nuestro trabajo sobre el amor, la lealtad, la constancia de los objetos y la intimidad, se ve acentuada por la estricta prescripción de ningún contacto social a partir de entonces. Así creamos una situación arbitraria que tiene mucho en común con una muerte. [pág. 151]

Esta cita se puede aplicar al analista tanto como al paciente. Damos por sentado que a lo largo de los años sufriremos la pérdida de innumerables personas que han sido nuestros compañeros en un íntimo y profundo intercambio emocional. Sin embargo, cuando perdemos el contacto con nuestros pacientes, la pérdida suele ser total y definitiva.

En un intento por separar el dolor evitable del inevitable de estas experiencias, sugiero que el impacto negativo se ve exacerbado por:

(1) Inhibición (concepciones implícitas de nuestro rol) de la capacidad para reconocer el profundo impacto que tienen algunas de nuestras pérdidas.

(2) Obstáculos, intrínsecos a la naturaleza de la relación analítica, para que podamos beneficiarnos del proceso de duelo normal.

(3) Otras formas de pérdida más sutiles que también son frecuentes en la vida profesional del analista. 
Nuestra formación no nos prepara adecuadamente para enfrentar dichas pérdidas. Parte de la razón es que los tratamientos que realizamos bajo supervisión generalmente se encuentran en sus fases iniciales, por lo que un análisis más profundo de la fase de terminación es poco frecuente. Sin embargo, esta causa, por más racional que parezca, no explica por completo porque estamos tan poco preparados para explorar nuestras experiencias repetitivas de pérdida de manera tal que nos permitan crecer a partir de las mismas. Una vez más, estoy de acuerdo con Coltart, quien sugiere que los analistas son reacios a examinar el paralelismo entre la finalización del tratamiento y la muerte por qué...

Incluso una persona madura, juiciosa y sensata puede sentir un temor cercano a lo supersticioso al hablar de la muerte, y esto ciertamente incluiría cualquier referencia comparativa a la muerte con un fenómeno tan frecuente como terminar un tratamiento en nuestra propia vida profesional. [pág. 148]

Comienzo este artículo sobre nuestras experiencias de pérdida con algunos comentarios sobre la situación del analista cuando el paciente muere durante el curso del tratamiento, ya sea por suicidio o por causas naturales. Por supuesto que los terapeutas no están solos al experimentar dichas muertes, pero aquí sugeriré algunas de las formas en que la muerte de un paciente en el análisis es una situación inusual. Para poder delimitar algunos de los obstáculos que puede encontrar el analista para llevar a cabo un duelo saludable, describo lo que creo que requiere el duelo normal.

Las muertes comprenden tan solo una pequeña parte de las pérdidas que sufrimos. Analizaré la experiencia que supone las terminaciones planificadas y las que se dan de forma abrupta, y por qué a menudo no podemos registrar su impacto por completo.

Pérdidas más profundas y sutiles que la muerte o partida real de un paciente abundan en nuestra vida profesional y creo que exacerban en gran medida nuestras experiencias de duelo agudo. Estos desmoralizadores crónicos incluyen pérdidas de dignidad, integridad y convicción, debido al impacto de aspectos culturales más amplios, como el cinismo sobre el análisis, ideas preconcebidas respecto al manejo del cuidado y un énfasis excesivo en las soluciones farmacéuticas a problemas emocionales. Eventualmente, esto puede conducir a una pérdida de implicación en el trabajo analítico o "burnout"(agotamiento). Sostengo que esta condición, extremadamente dolorosa, no es diferente a la depresión severa en sus efectos sobre la autoestima, el nivel de motivación, la creatividad, las actitudes sobre el futuro y la experiencia de llevar una vida con sentido.

Aunque gran parte de esta discusión podría considerarse bajo el foco de las reacciones de contratransferencia, sugiero que algunas de las dificultades para el analista en las situaciones que describo derivan de cómo se le ha enseñado a concebir su rol. Por lo tanto, estas 
experiencias dolorosas innecesariamente prolongadas son en parte producto de la cultura analítica (Buechler, 1988, 1992) y, por supuesto, también consecuencia de los problemas de carácter particulares y las experiencias de vida de cada profesional.

En una sección final de este trabajo, propongo algunas ideas sobre cómo podemos soportar nuestras pérdidas inevitables y necesarias y limitar la frecuencia y el impacto de las innecesarias.

\section{La muerte de un paciente}

Por supuesto que cada muerte tiene su propio significado personal e individual; pero siento que hay ciertas experiencias que los analistas suelen tener tras la muerte de un paciente, sea esta por suicidio o por causas naturales. Esto puede llegar a ser extremadamente doloroso y de alguna manera, extraño.

Si el analista asiste a un funeral, puede ponerse en contacto, muy probablemente por primera vez, con los familiares más cercanos del paciente. Puede ser muy difícil encontrar palabras que uno sienta como apropiadas, y sin embargo, se puede esperar de nosotros que seamos especialmente hábiles a nivel interpersonal para manejar esta delicada situación. Es posible tener la sensación de conocer íntima y profundamente a todas las partes afectadas $y$, sin embargo, es probable también que nunca se hayan conocido o hablado anteriormente. Los familiares y amigos, que afrontan su dolor, pueden sentirse incómodos ante la presencia del analista, cuyo papel en la vida del paciente pueden haber vivido con resentimiento durante mucho tiempo. Es posible que se culpe, de alguna manera, al analista por la muerte, o pueden sentir que durante años han sido juzgados por él, sin ninguna oportunidad de defenderse o explicarse. Los familiares y analista pueden estar soportando una enorme carga de sentimientos en conflicto, sin tener recuerdos positivos en común que facilitarían el poder relacionarse entre sí.

La paciente que mencioné previamente viene a mi mente de forma vívida. Era una mujer inolvidable que tocó muchas vidas con su determinación y espíritu vivaz, y sé que mi sensación de pérdida tiene su eco en muchos otros. Pero no estoy segura de que hubiesen podido entender la manera en que yo me sentía, cuán duramente ella había luchado por una vida mejor. Verla morir a una edad tan joven me causó un dolor indescriptible. A diferencia de otros que la lloraron, no tenía a nadie con quien realmente pudiera hablar sobre lo que sentía. Sus familiares y amigos no eran los míos, y me habría parecido inapropiado hablar sobre el tratamiento con ellos. Parte del duelo de un analista acontece en la más absoluta soledad. ${ }^{2}$ 


\section{Duelo Sano}

Antes de seguir discutiendo sobre la experiencia del analista ante la muerte de un paciente, me gustaría hacer un comentario respecto a la naturaleza del proceso de duelo. Freud, claro está, en "Duelo y melancolía" (1917), se esforzó por discriminar las emociones que normalmente acompañan a la pérdida de la condición patológica y depresiva. Al hacerlo, definió el duelo normal como un proceso de adaptación a la pérdida al cambiar gradualmente la catexis del objeto perdido a objetos nuevos y disponibles.

Desde otra perspectiva, los teóricos de la emoción (Izard, 1971, 1972, 1977) han definido la tristeza como un aspecto universal de la experiencia humana en respuesta a la pérdida. Se diferencia de la depresión, a la cual se considera una respuesta compleja más que una emoción básica, en que la depresión incluye la tristeza como componente, pero también tiene otros aspectos emocionales, dependiendo de la experiencia de vida de la persona. La tristeza es el dolor emocional que es temporalmente natural sentir cuando se sufre una pérdida o un golpe significativo en la vida. La depresión, por otro lado, generalmente implica una pérdida de autoestima, una constricción del rango de otras emociones y un empobrecimiento general en la calidad de vida.

No todos los teóricos conceptualizan la tristeza como una respuesta saludable, funcional y limitada respecto a la pérdida, ni tampoco conciben la depresión como su variante patológica. Algunos (por ejemplo, Bonime, 1982) ven ira en el núcleo de la depresión en lugar de tristeza, y entienden la "práctica" de la depresión como una forma de vida destructiva con una base caracterológica. El trabajo de Spiegel (1960, 1965, 1967, 1968, 1980) sugiere que las emociones específicas que podemos encontrar en la depresión de cualquier individuo en particular pueden diferir, por lo que siempre es clínicamente importante no hacer suposiciones sobre lo que "es" la depresión, e investigar cuidadosamente la naturaleza de la experiencia depresiva de cada paciente.

Pero para aquellos psicoanalistas interpersonales que ven la depresión como la versión patológica de la tristeza, se pueden distinguir los dos estados con mayor facilidad al observar sus efectos a lo largo del tiempo, ya que la tristeza cura y pasa, mientras que la depresión tiene un efecto disruptor en el funcionamiento de la persona. Arieti (1978) capta bien la diferencia entre tristeza y depresión:

Es común la tristeza que sobreviene al ser humano cuando un evento adverso golpea su precaria existencia o cuando la discrepancia resultante entre la forma en que es la vida y como podría ser se convierte en el foco de su febril reflexión. En algunas personas, dicha tristeza va y viene de forma repetida, en otras, tan solo de vez en cuando. Es dolorosa, 
dilata las acciones y generalmente cura, a menudo, pero no siempre, después de propiciar en quien lo padece una comprensión más profundiza de su situación y catalizar su maduración.

Menos común, pero también frecuente de forma suficiente como para constituir una importante preocupación psiquiátrica, es la tristeza que no disminuye con el paso del tiempo, que parece sobredimensionada en relación con el supuesto evento que la precipitó, o inapropiada, o sin guardar relación con ninguna causa discernible, o reemplazando una emoción más congruente. Este dolor se ralentiza, interrumpe o irrumpe en las acciones de uno; genera una sensación de angustia que puede tornarse difícil de contener; a veces tiende a expandirse implacable en una psique que parece poseer una capacidad ilimitada para experimentar dolor mental; a menudo reaparece nuevamente incluso después de una aparente mejoría. Este estado emocional es habitualmente conocido como depresión. [p. 3]

¿Qué nos permite, si somos caracterológicamente capaces de ello, llorar una pérdida y seguir viviendo fructíferamente? ¿Cuál es la forma en la que vivimos nuestras pérdidas que distingue el duelo normal de la depresión patológica? Gaines (1997) sugiere que el duelo sano abarca dos tareas: el desapego que enfatizó la teoría de las pulsiones de Freud, y la tarea igualmente importante de crear continuidad manteniendo y construyendo a partir de la conexión con el objeto interno. Crear continuidad es, como describe Gaines, un esfuerzo muy interpersonal.

Crear y mantener la continuidad no es algo que se alcance fácilmente. Para muchas personas es difícil mantener la imagen interna si no tienen la oportunidad de poder expresar en voz alta lo que sienten y compartirlo con otros. En parte, esto tiene que ver con el papel del lenguaje en la preservación de la memoria. Se sabe que tanto la expresión de sentimientos e imágenes en palabras como la repetición y el intercambio de esas narraciones con otros tiene un efecto positivo en la memoria. Además, el acto mismo de comunicar la propia experiencia de una relación perdida tiene el efecto de "darle vida", que es muy diferente de la contemplación interna. Las manifestaciones de aprecio del objeto perdido por parte de otras personas valida y enriquece la imagen interior del doliente solitario. Las observaciones de otros sobre la relación del doliente con el objeto perdido pueden añadir valor a la comprensión de en qué consistía la relación, qué significaba para el individuo y cómo es relevante para su vida ahora y en el futuro. La disponibilidad de otras personas con quienes compartir la conexión con el objeto perdido ayuda a los dolientes a llevar la relación al contexto actual, permitiendo que forme parte de sus vidas en curso. De manera muy importante, la construcción interna de objetos se basa en la construcción social compartida de esos objetos. [p. 559] 


\section{El Duelo irresoluble del analista}

Esta descripción del proceso interpersonal de duelo pone en evidencia lo que a menudo falta al analista cuando su paciente muere. Incluso si su personalidad le permite llevar a cabo un proceso de duelo saludable, el contexto interpersonal no lo facilita. Es posible que nunca pueda comunicarse con nadie sobre el paciente, lo que puede dificultar el mantener internamente su imagen interna. Sin la oportunidad de hablar sobre el paciente, especialmente a otros que también lo conocían, el analista solo cuenta con sus propios recursos en su esfuerzo por comprender lo que significa para él haberle conocido y perderle.

Pero creo que algunas de las expectativas integradas en nuestra cultura analítica respecto a nuestro rol como terapeutas interfieren aún más profundamente en el proceso esencial de duelo. Como analistas, somos parte de una cultura más amplia, pero también somos miembros de una subcultura analítica, con sus costumbres, mandatos y suposiciones particulares (Buechler, 1988, 1992). Ésta en ocasiones dicta de manera sutil, las reacciones emocionales consideradas "apropiadas" y aceptables para un profesional. Aunque es posible que no podamos formular conscientemente todas las "reglas", probablemente podríamos escuchar la presentación del caso de un colega y acordar si su implicación con el paciente parece "excesiva".

Siento que hay momentos en que estos mandatos coartan el proceso normal de duelo. Voy a descomponer este proceso en sus componentes, para sugerir los elementos específicos del proceso saludable normal que no están disponibles para nosotros como analistas.

Para sanar es necesario reconocer el hecho de la pérdida. La negación de la realidad y del significado de la pérdida interfieren en el duelo normal (véase, por ejemplo, Riviere, 1936, y su discusión sobre la relación entre la negación y la depresión). Por lo tanto, en cualquier medida que nuestra cultura analítica desanime a que nos veamos a nosotros mismos implicados personalmente en nuestra relación con los pacientes, puede generar dificultades para reconocer en nosotros mismos lo que la pérdida de un paciente significa a nivel personal. Cualquier tendencia de personalidad que podamos tener hacia el uso de la negación como defensa se podría ver acentuada en este contexto.

El duelo es complicado en presencia de una agresión no resuelta hacia el objeto antes de la pérdida. (Esto, por supuesto, fue explicado por Freud en su ensayo de 1917). En la medida en que, como analistas, procuramos contener respuestas emocionales negativas hacia el paciente, al menos hasta que parezca terapéuticamente apropiado compartirlas (Winnicott, 1949), aceptamos la carga de llevar nuestros sentimientos negativos no resueltos durante 
gran parte de tiempo del tratamiento. Si el paciente muere o termina el tratamiento abruptamente, nos quedaremos con todos esos sentimientos.

El proceso de duelo se promueve si uno tiene la posibilidad de escuchar lo que significó la pérdida para otros y así, de este modo, poder mantener la comunicación con otros que sufren a lo largo del tiempo. Esto permite generar la sensación de continuidad necesaria en relación con el objeto interno perdido (Gaines, 1997). En general, para el terapeuta todo esto es imposible debido a los límites y mandatos de nuestro rol. Del mismo modo, la ausencia de una comunicación continuada con alguien sobre lo que el paciente significó para uno puede complicar nuestra respuesta cuando finaliza el tratamiento. No tenemos la creencia de necesitar ningún proceso que fomente la continuidad, incluso parece como si esperáramos comportarnos como si los pacientes fueran intercambiables, y la pérdida de uno pudiese borrarse con la aparición de otro.

Un componente común del proceso de duelo es la identificación con el objeto perdido, como un intento de compensar la pérdida (Freud, 1933; Jacobson, 1971). Pero esto puede ser difícil para aquellos analistas que mantienen la distancia emocional como parte de su encuadre de trabajo.

La posición de duelo (dolosa) requiere la aceptación temporal de la pasividad, que podría ser difícil de integrar con las concepciones de algunos analistas respecto a su papel.

Por lo tanto, si consideramos el duelo desde la perspectiva de Gaines (1997), como un proceso de desapego del objeto, para dar cabida a nuevos lazos y, por otro lado, de continuidad, para preservar el significado de la conexión interna a lo largo del proceso, nuestro rol como analistas interfiere mucho más con la continuidad que con el desapego. Nuestro contexto interpersonal, que no incluye a nadie más que conozca al paciente, no es el más idóneo para mantener el vínculo interno. Nuestro rol exige cierta distancia, nos alienta a contener sentimientos negativos sobre el paciente y fomenta cualquier tendencia que tengamos a delimitar la percepción del significado que para nuestras vidas puede tener el fallecimiento de nuestro paciente. La jornada laboral, vivida en segmentos de cuarenta y cinco minutos, puede alentar la ilusión de que podemos "seguir adelante" emocionalmente cada vez que suena el timbre. El papel analítico también puede exacerbar la incomodidad que podría apaciguarse con la pasividad que requiere afrontar un duelo. Nuestra posición, de tener muchos otros pacientes con necesidades urgentes, parece justificar, incluso ennoblecer, seguir adelante. Esto refuerza cualquier mecanismo defensivo en nosotros que pueda tender a negar el impacto de la pérdida. Es como si nuestro rol hiciera parecer posible que podamos "omitir" parte del proceso normal de duelo humano. Si tenemos caracterológicamente alguna tendencia en esta dirección, el rol las fomenta. 
Mi experiencia analizando, supervisando y hablando informalmente con analistas a lo largo de los años sugiere que muchos de nosotros hemos sufrido la muerte de pacientes sin hacer un esfuerzo activo para procesar la pérdida de manera significativa. No tenemos rituales institucionalizados para afrontar estas pérdidas. Es como si el hecho de que la persona fuera un "paciente" hace que el ritual sea innecesario. Sería poco probable, si un colega, amigo o pariente muriera o se fuera de forma permanente, esperar que "sigamos adelante" sin llorar. Pero debido a que, en un sentido fundamental, nuestro rol alienta la negación del impacto personal de nuestra relación con los pacientes, también negamos el significado personal de su muerte o partida.

Hay complicaciones adicionales si la muerte de un paciente es el resultado de un suicidio. No hay duda de que el suicidio puede perseguir al analista de por vida. Entre otros sentimientos, puede surgir la sensación de culpabilidad (creer que uno podría haberlo evitado). La mayor parte de los autores que escriben sobre el duelo sugieren que la culpa, o cualquier otro factor que pueda disminuir la autoestima, interfiere en el proceso de duelo normal (Arieti y Bemporad, 1978). Tal vez, al igual que los familiares y amigos del paciente, es probable que el analista recorra mentalmente cada interacción reciente con el fallecido en busca de pistas sobre el estado desesperado del paciente que el analista pudo haber pasado por alto. Pero a diferencia de amigos y parientes, el analista es, supuestamente, un experto en interpretar el comportamiento humano. Desafortunadamente, por supuesto, nuestra experiencia no garantiza que siempre podamos predecir lo que un paciente puede hacer. Pero, sobre todo si al mirar hacia atrás, vemos que no estuvimos dispuestos a ofrecer más al paciente, podemos sentir una particular sensación de responsabilidad y culpa.

\section{Terminaciones, planificadas y abruptas}

Incluso cuando el final del tratamiento está bien planificado implica varios tipos de pérdidas para el analista: la pérdida de un compañero en una relación significativa, una pérdida económica y la pérdida de una reflexión, es decir, alguien que podría ayudar al analista a evaluar su propio progreso profesional. La literatura sobre la finalización del tratamiento (por ejemplo, Ferenczi, 1927; Freud, 1937; Reich, 1950) trata principalmente sobre el momento e impacto para el paciente y es menos probable que preste atención a las posibles pérdidas que tiene para el terapeuta.

Al final creo que lo que todos hacemos es, desde mi perspectiva, un desfile de viejos pacientes. Me encantaría saber qué les sucedió, aunque solo fuera por curiosidad natural. Por supuesto, el nuestro no es el único campo en el cual quien lo ejerce trabaja hacia su propia caducidad. Pensemos en la crianza o incluso en la paternidad. Pero para aquellos de 
nosotros que sentimos que el contacto posterior al tratamiento debe ser muy limitado, existe una particular sensación de pérdida al finalizar un tratamiento. No poder saber nunca más lo que pasó con alguien que has conocido tan íntimamente es una pérdida increíble. Sin embargo, es algo habitual en nuestra vida profesional. Es parte de nuestro trabajo.

Pero hasta aquí tan solo hemos arañado la superficie de la cuestión si pensamos en las pérdidas que soportan los analistas. Además de las finalizaciones de tratamiento planificadas, hay terminaciones abruptas. Apostaría a que ningún analista completa su carrera sin encontrarse al menos con un paciente misterioso que se va en medio del trabajo, dando razones circunstanciales pero insuficientes. Freud (1905) nos dio un ejemplo de su expresión abierta de dolor y frustración en respuesta a la partida abrupta de su paciente Dora. Como estudiante de posgrado, recuerdo haber visto una entrevista grabada en la que "una eminente autoridad", hablaba sobre terminaciones inexplicables. Decía que "partes" de estos pacientes siempre "se quedaron con él". Para el analista, la historia nunca termina; permanece inacabada. Siempre puede preguntarse si podría haber hecho algo diferente para hacer posible que el trabajo continuase. Además de sentirse responsable de los "errores" que pudo haber cometido, es posible que no pueda liberarse por completo de como concibió la situación en aquel entonces.

Quienes trabajan con niños experimentan, a menudo, un agudo dolor cuando los padres retiran del tratamiento al niño tan pronto como comienza a percibirse cierto progreso (desde el punto de vista del terapeuta). El triángulo padre/madre-hijo/a-analista es intrínsecamente complicado, y no voy a expresar sus dificultades aquí, pero puedo dar fe del tremendo dolor, particularmente evidente en los jóvenes terapeutas más idealistas, que pueden identificarse de forma profunda con sus pacientes infantes y solo les queda observar su retorno a situaciones familiares abusivas o negligentes, sin poder hacer nada, a menos que sean capaces de presentar pruebas que justifiquen su intervención.

Mis comentarios, sobre la finalización abrupta del tratamiento, tienen la intención de evocar, recuerdos y memorias y no tratar de definir las situaciones. Cada circunstancia tiene su propio impacto particular, pero a menos que nuestra formación y nuestras experiencias posteriores nos animen al auto análisis de cómo nos han afectado y afectan estas situaciones en particular, creo que podrían terminar contribuyendo a un estado corrosivo de agotamiento (burnout) que discuto más adelante. Aunque el tratamiento y la partida de cada paciente tiene su propio significado, si un analista reflexiona sobre su experiencia, es posible que descubra que tiene una forma característica de responder a las terminaciones abruptas. Para algunos de nosotros la autocensura y un fuerte sentimiento de culpa pueden predominar, mientras que, para otros, puede prevalecer la vergüenza y un doloroso 
sentimiento de insuficiencia. Otros pueden experimentar principalmente rabia dirigida hacia el paciente. A menos que encontremos una manera de enfrentar y afrontar verdaderamente estas experiencias, siento que pueden afectar a nuestro compromiso con el trabajo y a nuestra fe en nosotros mismos.

\section{Pérdidas basadas en la cultura y "Burnout"}

Sin demasiado esfuerzo, la mayoría, podríamos describir pérdidas sufridas como resultado de cuestiones administrativas. En muchos casos, nosotros y nuestros pacientes podemos descubrirnos en posiciones insostenibles. Debemos colaborar con un sistema legal-sanitario que a menudo no muestra respeto por los valores básicos de nuestra profesión o corremos el riesgo de perder, para nuestros pacientes, el reembolso que necesitan. El hecho de que el tratamiento se reembolse o no, puede llegar a depender de la habilidad verbal del "proveedor" del servicio, que debe expresar la "enfermedad" del paciente en términos aceptables para la empresa aseguradora. Las pérdidas para todos los interesados son demasiado numerosas para enumerarlas una por una, pero muchas veces incluye la pérdida de dignidad tanto para el paciente como para el analista. Independientemente de la decisión que tome el analista, ya sea "jugar el juego" e intentar acertar con las palabras que permitirían a su paciente acceder a un reembolso, o simplemente informar de los hechos u optar por salir del sistema, paciente y analista se pueden llegar a ver obligados a desempeñar roles potencialmente problemáticos entre ellos. La habilidad verbal del analista, la voluntad de estirar la verdad, las actitudes respecto al sistema, el ingenio para encontrar tiempo para el papeleo y cosas similares no deberían ser lo que determine si el paciente recibe o no un reembolso; pero estos y otros factores igualmente absurdos a menudo marcan la diferencia.

Muchos analistas acuden a consulta o supervisión por haber perdido el entusiasmo en el campo profesional, en parte debido al clima actual donde impera la búsqueda rápida de resultados. Los más jóvenes, recién llegados al campo, pueden llegar a plantearse si ha sido inteligente elegir esta profesión. ¿Habrá análisis dentro de diez, veinte o treinta años? ¿Cómo es posible llevar a cabo prácticas decentes si cada vez más, la mentalidad popular, apoyada por las autoridades institucionales, aspira a que sus problemas profundamente arraigados sean erradicados gracias a una píldora mágica, o en ocho sesiones de cuarenta y cinco minutos? Los analistas, de mediana edad, ven que sus ingresos y oportunidades para usar sus habilidades están disminuyendo, cuando esperaban alcanzar el punto más alto a nivel profesional y sienten que ya es demasiado tarde para cambiar de profesión. Los mayores miran con creciente consternación lo que le ha sucedido al campo al que han dedicado el trabajo y el esfuerzo de sus vidas. 
Hasta ahora, he descrito la experiencia de pérdidas por muertes, terminaciones planificadas y abruptas, y el clima desmoralizador. De forma acumulativa, estas experiencias pueden, a mi parecer, contribuir a lo que a menudo se llama "burnout"(agotamiento) (Cooper, 1986). El agotamiento es sintomático de la mayor pérdida de todas: la pérdida de la fe en la profesión y en uno mismo. El analista agotado ya no cree en el valor de lo que hace para ganarse la vida. Siente que, por el motivo que sea, económico o de cualquier otro tipo, debe tirar hacia adelante y seguir con los tratamientos, pero se ha convertido en un ejercicio vacío. Creo que las pérdidas acumuladas son una fuente importante de agotamiento (burnout), aunque otros factores, como las tensiones propias del trabajo y problemas de personalidad particulares del profesional, pueden también jugar un papel importante.

Parece ser que, en el agotamiento, el hecho de pensar que nuestro trabajo tuviese menos trascendencia de la que tiene, puede ofrecer cierto consuelo o alivio de las experiencias repetitivas de estrés, dolor y pérdida. Si lo que estoy haciendo no tiene sentido, entonces tal vez perder pacientes y el clima desalentador no producirá tanto dolor. A nivel inconsciente, el cinismo ofrece una ilusión de protección contra el dolor. Es mejor replegarse uno mismo que ser asaltado continuamente por la pérdida. Por supuesto, esta no es una buena solución, y el analista agotado, quemado, sufre la mayor pérdida posible: la pérdida de la fe en su valía y en lo que puede lograr.

Diría que, en general, la mayor parte de las pérdidas que he descrito evocan una tristeza limitada en el tiempo. La reacción de burnout, de agotamiento, creo que se encuentra más próxima a la depresión que a la tristeza (ver la descripción anterior de la diferencia entre las dos). El agotamiento o burnout lleva aparejado una clara disminución de la autoestima y es probable que se generalice y se prolongue a lo largo del tiempo, en lugar de ser una respuesta transitoria. ¿Por qué mientras que algunos nos agotamos otros son capaces de llevar una vida profesional larga y próspera? Como en cualquier caso de depresión, la estructura de personalidad de quien la padece es clave (Jacobson, 1971). Pero también creo que aquellos de nosotros que estamos implicados en la formación tenemos la obligación de hablar y escribir abiertamente sobre nuestras propias experiencias de pérdida, para que los analistas más jóvenes se sientan libres de reflexionar y si es necesario obtener ayuda pronto para poder afrontar las pérdidas que enfrentarán en su vida profesional. Si nos reservamos únicamente para nosotros mismos lo que significa a nivel personal perder pacientes de una forma u otra, año tras año, no seremos capaces de ofrecer a nuestros colegas más jóvenes una oportunidad para que puedan abordar estos temas de forma abierta. 


\section{La pérdida bien transmitida}

Aunque en nuestro trabajo, las experiencias repetidas de pérdida son inevitables, podemos, aprender a soportarlas de una mejor manera. Además de permitirnos a nosotros mismos ser conscientes de la importancia de las pérdidas, de modo que la negación no complique el proceso de duelo, podríamos recurrir, con más frecuencia, a nuestros colegas, para convertirlas en experiencias compartidas. Podemos procurar reflexionar respecto al potencial que tienen para promover nuestro crecimiento personal y profesional.

Espero que al escribir sobre los posibles usos positivos de estas experiencias no se me malinterprete como minimizando su importancia. Pero sí siento que pueden contribuir a la vida emocional del analista. Por supuesto que no estoy sugiriendo que se deban procurar pérdidas como recurso, pero debemos estudiar de forma conjunta cómo se podrían afrontar de tal manera que el analista evite caer en el agotamiento o burnout.

Cada año que pasa valoro más profundamente la afirmación de Sullivan de que las personas somos más humanos que cualquier otra cosa. Al ser partícipe de los momentos más dolorosos en la vida de los demás, he llegado a darme cuenta cómo el comportamiento aparentemente más inexplicable puede, en el fondo, ser una compleja expresión de un duelo que no deja de ser algo bastante básico y universal. El analista siempre está cerca de las pérdidas, en ocasiones intensas, que forman parte de la vida cotidiana. La pérdida de seres queridos, de la salud, de la fe, de los ideales, de los sueños. Experimenta la pérdida de forma vicaria, a través de las experiencias de sus pacientes, pero también a través de sus propias pérdidas.

Este mayor nivel de conciencia de la omnipresencia de la pérdida no es tan solo una carga. También puede ser un gran incentivo para saborear cada momento de la vida. Puede, potencialmente, enseñar las mismas lecciones que ofrece cualquier experiencia cercana a la tragedia; mejorar la capacidad para percibir los pequeños milagros de la vida, los placeres cotidianos que tan fácilmente se dan por sentados.

La tristeza es una emoción que potencialmente nos une. Me invita a comprender lo que inicialmente me resulta extraño. Cuando comencé a trabajar, me asignaron el caso de un paciente que era miembro del Partido Nazi Americano. Sus creencias eran especialmente repugnantes para mí e inicialmente, tenía dudas sobre si iba a ser capaz de tratarlo. Pero algo me llevó a escuchar a este paciente más allá de su propio discurso y al oír la historia de su infancia, de una tristeza verdaderamente insoportable, hallé un ser humano con el que si podía conectarme.

Podía sentir pena por sus pérdidas, por la tierna y sana infancia que jamás podría tener. Pude entender cómo todo aquello pervirtió su desarrollo y lo convirtió en la persona amargada 
que era, aunque también sabía que nunca abrazaría la "filosofía" que él desarrolló. Gradualmente llegó a rechazar esa filosofía junto con los esfuerzos defensivos de dominación absoluta y de anhelo de superioridad que la definían. Pudimos entender algunos de los complejos determinantes de su posición política. Quedó claro que afiliarse a un partido fue más que una mera respuesta a una infancia severamente abusiva, y no puede explicarse de una manera simple. Sin caer presa de cualquier forma de reduccionismo, siento que ser capaz de empatizar con el genuino dolor de mi paciente ante alguna de sus primeras pérdidas me permitió conectar con aspectos de su humanidad y gracias a ello fui capaz de soportar estar con él, el tiempo suficiente como para poder conocerlo y ayudarlo.

La tristeza finalmente pasa, lo que nos permite volver a la lucha, pero nos familiariza de un modo más profundo con lo que significa ser humano. Nos une, unos a otros, y mejora nuestra capacidad para apreciar los momentos felices. Da perspectiva a las vicisitudes ordinarias y extraordinarias de la vida. Como analista, es necesario desarrollar una fuerte tolerancia a la tristeza y a la pérdida, ya que estarán siempre cerca.

Cada vez que giro en una esquina "veo" a la paciente que mencioné anteriormente, que murió hace años. Me doy cuenta de la frecuencia con que esto me ha sucedido a pesar de los años transcurridos desde su muerte. Al principio pensé que sería un fenómeno fugaz, una ilusión que expresaba cuánto echo de menos su humor y su coraje. Entiendo que estos momentos reflejan de forma significativa aspectos personales de mi relación continua con la pérdida. Pero durante esos breves instantes, ella está aquí conmigo nuevamente, y no solo viva, sino bien, caminando, fuera de su silla de ruedas. La fantasía me da un descanso momentáneo del peso que me genera la triste realidad. Y luego la persona en la calle se acerca, y me doy cuenta de que no es mi paciente. El dolor de su pérdida no es menor ahora que cuando murió, y mis sentimientos de pesar por no haber tenido más tiempo no han cambiado. Lo que es diferente es que tras años de autorreflexión veo más claramente algunos de los significados personales de su vida y de su muerte. Traigo esta reflexión conmigo cada vez que lloro por su pérdida y la experiencia acumulada por los años me ha enseñado algo sobre cuánto contribuyó a mi crecimiento personal y profesional. En la actualidad, su recuerdo ilumina muchos de mis momentos clínicos y no clínicos, y esto hace que mi aprecio por ella sea cada vez mayor. Cuando su recuerdo me ayuda de alguna manera, el equilibrio de lo que obtuve de ella en contraposición con lo que sufrí, cambia. Ahora sé mucho más sobre lo que significó y aún significa haberla conocido, tanto a nivel profesional como personalmente, y de qué modo me gustaría ser capaz de usar lo que me hizo crecer. La tristeza está haciendo su trabajo esencial, uniéndome más firmemente a la vida. 


\section{REFERENCIAS}

Arieti, S. (1978). The basic questions and the psychological approach. In: Severe and Mild Depression, S. Arieti \& J. Bemporad. New York: Basic Books, pp. 3-10.

Arieti, S. \& Bemporad, J. (1878). Severe and Mild Depression. New York: Basic Books.

Bonime, W. (1982). Psychotherapy of the Depressed Patient. Contemp. Psychoanal. 18:173-189

Buechler, S. (1988). Joining the Psychoanalytic Culture. Contemp. Psychoanal. 24:462-469

Buechler, S. (1992). Stress in the personal and professional development of a psychoanalyst. J. Am. Acad. Psychoanal. Dyn. Psychiatr., 20:183-191.

Buechler, S. (2012). Still Practicing: The Heartaches and Joys of a Clinical Career. New York, NY: Routledge.

Buechler, S. (2015). Marcando la diferencia en las vidas de los pacientes. Experiencia emocional en el ámbito terapéutico. Madrid: Ágora Relacional. [V. original: Making a difference in patients' lives. New York: Routledge, 2008]

Buechler, S. (2018a). Valores de la Clínica. Emociones que guían el tratamiento psicoanalítico. Madrid: Ágora Relacional [v. Original: Clinical values: Emotions that guide psychoanalytic treatment. Hillsdale, NJ: The Analytic Press, 2004]

Buechler, S. (2018b). Psychoanalytic Reflections. Training and Practice. IPBooks.

Buechler, S. (2019a). Comprendiendo y tratando a pacientes en psicoanálisis clínico. Lecciones desde la literatura. Madrid: Ágora Relacional. [V. original: Understanding and treating patients in Clinical Psychoanalysis. Lessons from literature. New York: Routledge, 2015]

Buechler, S. (2019b). Psychoanalytic approaches to problems in living. Addressing life's changes in clinical practice. New York: Routledge.

Coltart, N. (1996). The Baby and the Bathwater. Madison, NJ: International Universities Press.

Cooper, A. M. (1986). Some Limitations on Therapeutic Effectiveness: The "Burnout Syndrome" in Psychoanalysts. Psychoanal Q. 55:576-598

Ferenczi, S. (1927). The problem of the termination of the analysis. In: Final Contributions to the Problems and Methods of Psychoanalysis, ed. M. Balint. New York: Basic Books, pp. 77-86.

Freud, S. (1905). Fragment of an analysis of a case of hysteria. Standard Edition, 7:3-122.

Freud, S. (1917). Mourning and melancholia. Standard Edition, 14:237-258.

Freud, S. (1933). New introductory lectures on psychoanalysis. Standard Edition, 22:3-184.

Freud, S. (1937). Analysis terminable and interminable. Standard Edition, 23:211-253.

Gaines, R. (1997). Detachment And Continuity. Contemp. Psychoanal. 33:549-571

Izard, C. E. (1971). The Face of Emotion, New York: Meredith Corporation.

Izard, C. E. (1972). Patterns of Emotions. New York: Academic Press.

Izard, C. E. (1977). Human Emotions. New York: Plenum Press. 
Jacobson, E. (1971). Depression: Comparative Studies of Normal, Neurotic, and Psychotic Conditions. New York: International Universities Press.

Reich, A. (1950). On the Termination of Analysis. Int. J. Psycho-Anal. 31:179-183

Riviere, J. (1936). A Contribution to the Analysis of the Negative Therapeutic Reaction. Int. J. PsychoAnal. 17:304-320

Spiegel, R. (1960). Communication in the psychoanalysis of depressions. In: Psychoanalysis and Human Values, ed. J. H. Masserman. New York: Grune \& Stratton, pp. 209-222.

Spiegel, R. (1965). Communication with Depressive Patients. Contemp. Psychoanal. 2:30-34

Spiegel, R. (1967). Anger and acting out: Masks of depression. J. Am. Acad. Psychoanal. Dyn. Psychiatr., 21:597-606.

Spiegel, R. (1968). Supervisory Collaboration in the Treatment Strategy for Masked Depression. Contemp. Psychoanal. 5:57-61

Spiegel, R. (1980). Cognitive aspects of affects and other feeling states with clinical applications. J. Am. Acad. Psychoanal. Dyn. Psychiatr., 8:591614.

Winnicott, D. W. (1949). Hate in the Counter-Transference. Int. J. PsychoAnal. 30:69-74

Original recibido con fecha: 17-11-2020 Revisado: 15/3/2021 Aceptado: 30/03/2021

NOTAS:

${ }^{1} \mathrm{~N}$. de. T: Los años remiten a las ediciones originales, que se corresponden en las referencias con las indicadas como 2012, 2015, 2018a, 2019a. La autora escribe esta introducción al incluir la revisión de este trabajo en su obra "Reflexiones Psicoanalíticas" (2019b)

2 Por los muchos años en que estas personas han atenuado esa soledad, me gustaría agradecer a los miembros de mi grupo de supervisión: los Dres. Mark Blechner, Richard Gartner, John O'Leary, Allison Stern Rosen y Robert Watson. 